\title{
Thermal Conductivity in Nanoporous Gold Films during Electron-Phonon Nonequilibrium
}

\author{
Patrick E. Hopkins, ${ }^{1}$ Pamela M. Norris, ${ }^{1}$ Leslie M. Phinney, ${ }^{2}$ Steven A. Policastro, ${ }^{3}$ and Robert G. Kelly ${ }^{3}$ \\ ${ }^{1}$ Department of Mechanical and Aerospace Engineering, University of Virginia, Charlottesville, VA 22904-4746, USA \\ ${ }^{2}$ Engineering Sciences Center, Sandia National Laboratories, Albuquerque, NM 87185, USA \\ ${ }^{3}$ Department of Materials Science and Engineering, University of Virginia, Charlottesville, VA 22904-4746, USA
}

Correspondence should be addressed to Pamela M. Norris, pamela@virginia.edu

Received 29 November 2007; Accepted 28 May 2008

Recommended by Claude Estournes

\begin{abstract}
The reduction of nanodevices has given recent attention to nanoporous materials due to their structure and geometry. However, the thermophysical properties of these materials are relatively unknown. In this article, an expression for thermal conductivity of nanoporous structures is derived based on the assumption that the finite size of the ligaments leads to electron-ligament wall scattering. This expression is then used to analyze the thermal conductivity of nanoporous structures in the event of electronphonon nonequilibrium.
\end{abstract}

Copyright (C) 2008 Patrick E. Hopkins et al. This is an open access article distributed under the Creative Commons Attribution License, which permits unrestricted use, distribution, and reproduction in any medium, provided the original work is properly cited.

\section{INTRODUCTION}

The continued reduction in characteristic length scales of nanodevices has driven the need to understand the thermal characteristics of low-dimensional structures [1]. For example, the quasi-one-dimensional geometry of nanowires makes them ideal for applications such as field effect transistors (FETs), which are based on the transport of charge. However, the heat generation from the charge transport and subsequent thermal management of these FETs pose an ever-growing problem in further development and design due to thermal phenomena that arise as a result of the reduction of the characteristic lengths of the materials [2]. With the use of nanowires in FETs, a significant reduction in thermal conductivity results [3], which can drastically limit operational frequencies and powers since the removal of the generated heat is reduced compared to bulk. In addition, an increase in operational frequencies and powers in FETs can result in a large electric field experienced by the electrons which can throw the electrons out of equilibrium with the lattice resulting in another form of thermal resistance [4]. In this report, thermal processes in nanoporous gold films are considered. The nanoporous gold is essentially a random matrix of Au nanowires that exhibit a reduction in thermal conductivity due to scattering at the wire surfaces. During nonequilibrium electron-lattice processes, these scattering events must be considered to accurately predict heat flow through the structure. The rate of energy loss by an electron system out of equilibrium with its lattice is measured in nanoporous Au [5-7] with a pumpprobe transient thermoreflectance (TTR) technique [8].

\section{THERMAL CONDUCTIVITY REDUCTION IN NANOWIRES}

The electron lattice nonequilibrium, which drives electronphonon coupling, has been the focus of several studies [8$20]$ and recently this interest has extended to nanowires [2123]. In the case of bulk materials, at room temperature, the resistance to electron transport is dominated by phonon scattering. However, when the characteristic length, $l$, of a material is on the order of the mean free path of the electrons, $\lambda$, then the resistance becomes affected by scattering of electrons at surfaces. In the case of the nanoporous $\mathrm{Au}$, the electron-surface scattering has been attributed to electronic coupling of adsorbates to the conduction electrons which gives rise to the change in resistance exploited in several applications [7].

This subsequent reduction in electron system thermal conductivity (from here on will simply be referred to as 


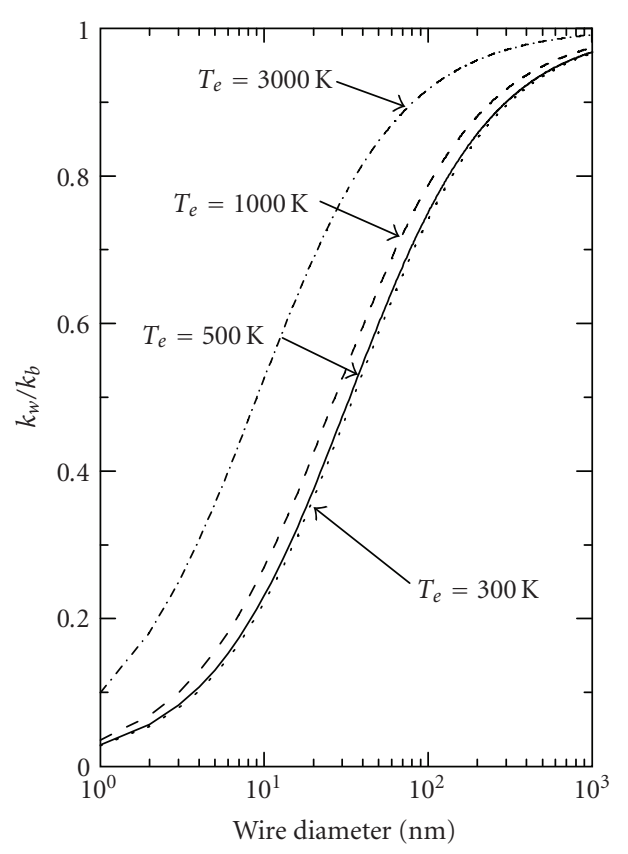

(a)

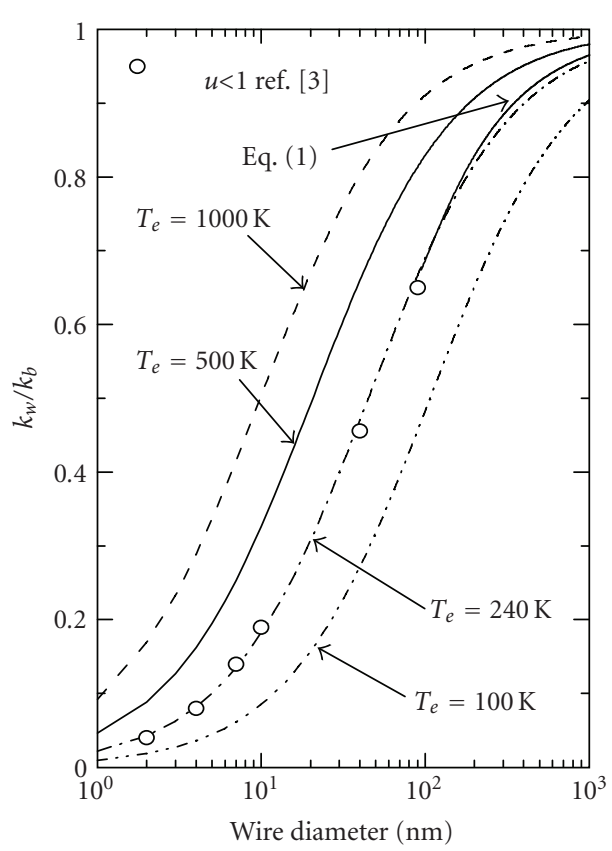

(b)

FIGURE 1: Ratio of wire thermal conductivity, (2), to bulk thermal conductivity versus wire diameter for several different electron temperatures (a) during electron-phonon nonequilibrium when $T_{p}=300$ and (b) when $T_{e}=T_{p}$. The calculations shown when $T_{e}=T_{p}=240 \mathrm{~K}$, corresponding to an electron mean free path of $45 \mathrm{~nm}$, agree well with previous results using $\lambda=41.7 \mathrm{~nm}$.

the thermal conductivity or conductivity since the focus of this work is metal systems) that is associated with surface scattering is given by [3]

$$
\frac{k_{w}}{k_{b}} \approx 1-\frac{3}{4 u}+\frac{3}{8 u^{3}}
$$

where $k_{w}$ is the reduced thermal conductivity, $k_{b}$ is the conductivity of the corresponding bulk material, and $u$ is the ratio of the wire diameter, $d$, to the electron meanfree path in the wire, $\lambda$. In the case of nanoporous $\mathrm{Au}$, the wire diameter in (1) refers to the average ligament size. The corresponding bulk conductivity can be calculated from kinetic theory by $k_{b}=C_{e} v_{F} \lambda / 3$, where $C_{e}$ is the electronic heat capacity, which at the temperatures of interest $\left(T_{e}<\right.$ $5000 \mathrm{~K})$ can be calculated by $C_{e}=y T_{e}$ with $\gamma$ being the Sommerfeld constant $[20,24]$, and $v_{F}$ is the Fermi velocity. The mean free path of the electrons in bulk is dominated by electron and phonon scattering, so $\lambda$ can be estimated by $\lambda=v_{F} \tau$, where $\tau$ is calculated with Matthiessen's rule by taking into account electron-electron scattering, $1 / \tau_{e e}=$ $A T_{e}^{2}$, and electron-phonon scattering, $1 / \tau_{e p}=B T_{p}$, where $\tau_{e e}$ is the electron-electron scattering time, $\tau_{e p}$ is the electronphonon scattering time, $T_{e}$ is the electron temperature, $T_{p}$ is the lattice (or phonon) temperature, and $A$ and $B$ are scattering coefficients [25-27] that are weakly dependent on temperature in $\mathrm{Au}$ [28]. Matthiessen's rule assumes that there are multiple physically distinguishable sources of scattering (e.g., electron-electron or electron-phonon), and the presence of one scattering mechanism does not alter the way in which the other mechanisms function [29].
Equation (1) is a result of an exponential integral expansion that is valid as long as $u>1$. Predicting the reduction in wire thermal conductivity requires significantly more consideration when $u<1$ [3]. However, by considering electron-boundary scattering in $k_{b}, k_{w}$ can be easily estimated for all $u$. When calculating $\lambda$ in a nanowire, the effects of electron-boundary scattering can be incorporated via Matthiessen's rule. This can be estimated as $1 / \tau_{B D}=v_{F} / d$ assuming complete diffuse scattering [30]. Note that $\tau_{B D}$ is considered temperature independent [22]. Therefore, the conductivity of a nanowire can be expressed as

$$
k_{w}\left(T_{e}, T_{p}, d\right)=\frac{1}{3} v_{F}^{2} \frac{\gamma T_{e}}{A T_{e}^{2}+B T_{p}+\left(v_{F} / d\right)},
$$

which is dependent on electron and phonon temperatures and the wire diameter, and therefore can be used to examine thermal conductivity of nanowires in the event of electron-phonon nonequilibrium heating. Figure $1(\mathrm{a})$ shows the ratio $k_{w} / k_{b}$ as a function of Au wire diameter for various electron temperatures. In these calculations, the effects of electron phonon nonequilibrium are studied, therefore $T_{p}$ is assumed as $300 \mathrm{~K}$. In Figure 1(b), the thermal conductivity is calculated as a function of wire diameter for various electron temperatures assuming $T_{e}=T_{p}$. These results are compared to calculations using (1) when $u>1$ and simulation results from Au nanowires assuming $\lambda=41.7 \mathrm{~nm}$ when $u<1$. Note that the results from [3] for $u<1$ and (1) for $u>1$ agree well with (2) over the entire range of wire diameters when $T_{e}$ equals $240 \mathrm{~K}$, which corresponds to a $\lambda$ of $45 \mathrm{~nm}$ assuming no boundary scattering. Figure 2 presents the electron 


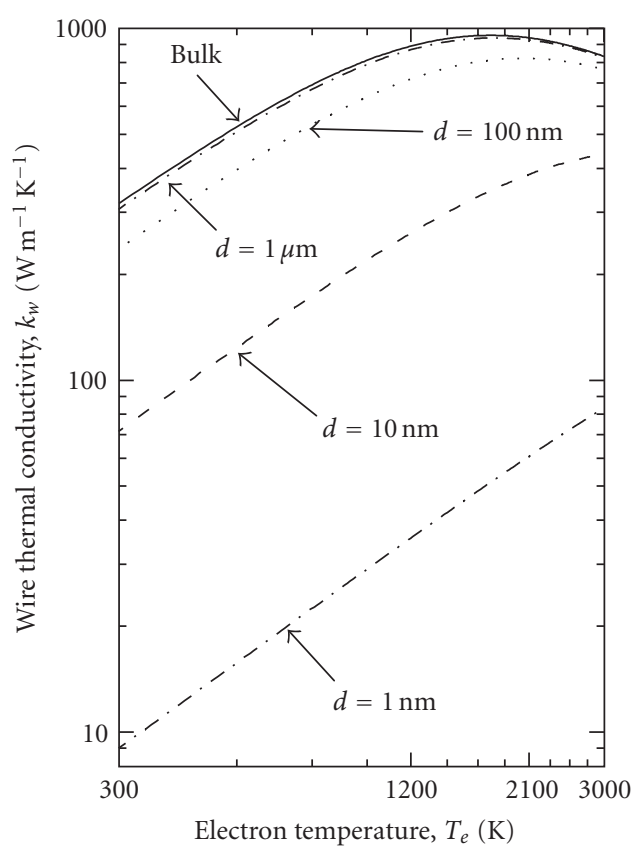

(a)

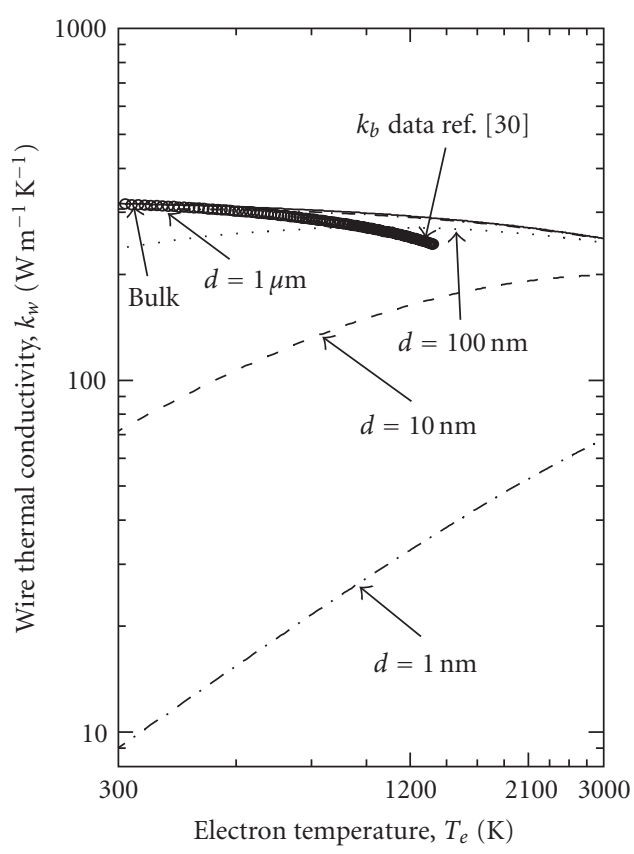

(b)

FIGURE 2: Wire thermal conductivity versus electron temperature for several different wire diameters (a) during electron-phonon nonequilibrium when $T_{p}=300$ and (b) when $T_{e}=T_{p}$. In the case of a bulk, (2) used to predict wire diameter agrees well with experimental data of thermal conductivity data taken on bulk Au at relatively low temperatures.

thermal conductivity as a function of temperature for several different Au wire diameters. Similar to Figure 1, Figure 2(a) assumes an electron-phonon nonequilibrium with $T_{p}=$ 300, and Figure 2(b) assumes $T_{e}=T_{p}$. Conductivity data on bulk $\mathrm{Au}$ [31] in Figure 2(b) agrees well with (2) when $d \gg \lambda$. The nonequilibrium effects on thermal conductivity are apparent by comparing Figures $1(\mathrm{a})$ and 2(a) to Figures 1(b) and 2(b). Obviously, at high temperatures, electronphonon nonequilibrium will result in a higher conductivity than equilibrium. In the nonequilibrium case, the lattice is colder than the equilibrium case which results in less electron-phonon scattering thereby increasing $\lambda$. Note in the case of very small $d$, the equilibrium and nonequilibrium situations are the same since $\lambda$ is restricted by electronboundary scattering.

\section{THERMAL CONDUCTIVITY REDUCTION IN NANOPOROUS MATERIALS}

Although (2) gives the reduction in thermal conductivity due to boundary scattering, there will still be a further reduction in conductivity due to the porous nature of the film. As the electrons are conducting through the Au structure, the electron-boundary scattering is significantly increased since the "nanowires" that comprise the Au mesh cannot be considered as $1 \mathrm{D}$ conducting channels. In actuality, the path of the conducting electrons has several random turns and kinks as a result of the fabrication process. This further conductivity reduction can be estimated by considering the percent porosity of the nanostructure $[32,33]$. By treating the pores as randomly sized spheres, the reduction in thermal conductivity of the porous film can be calculated with the Bruggeman assumption [34, 35] and estimated by

$$
\frac{k_{p}}{k_{w}}=(1-f)^{3 / 2},
$$

where $f$ is the porosity, $k_{w}$ is the thermal conductivity of the solid material, in the case of the nanoporous $\mathrm{Au}$, the reduced thermal conductivity due to the ligament size being on the order of $\lambda$ must be used, and $k_{p}$ is the reduced thermal conductivity of the nanoporous Au. Combining (2) and (3) results in an expression for electron thermal conductivity in nanoporous metal composites. Figure 3 gives the calculations for conductivity of nanoporous gold as a function of electron temperature for four different porosities, $f$, of $0 \%, 15 \%, 30 \%$, and $50 \%$ and three different wire diameters, $d$, of $10 \mathrm{~nm}, 100 \mathrm{~nm}$, and $1 \mu \mathrm{m}$ assuming $T_{p}=300 \mathrm{~K}$. As expected from (3), an increase in porosity continues to reduce the overall thermal conductivity of the nonporous Au. During electron-phonon nonequilibrium, a change in the thermal conductivity would change the time it takes for the electrons and phonons to equilibrate. If the conductivity is significantly reduced, the energy density of the electron system would remain large near the heat source causing a change in the thermalization time $[14,36]$. The relationship between change in electron temperature and thermal conductivity and electron-phonon coupling is given in the two-temperature model (TTM) [9]. Therefore, the effect that the change in electron temperature has on wire 


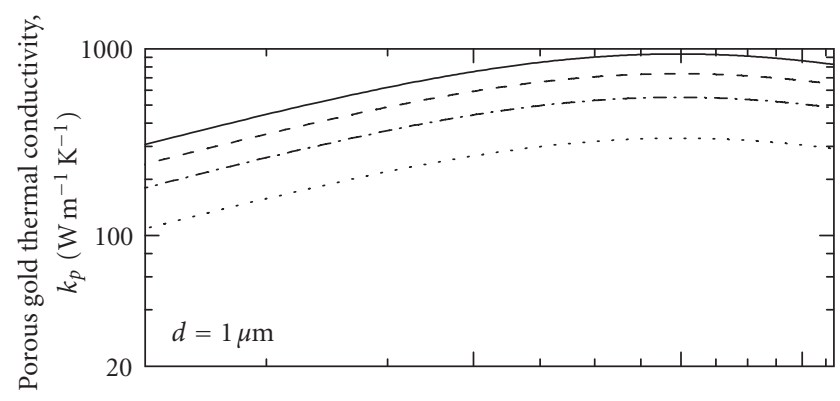

(a)

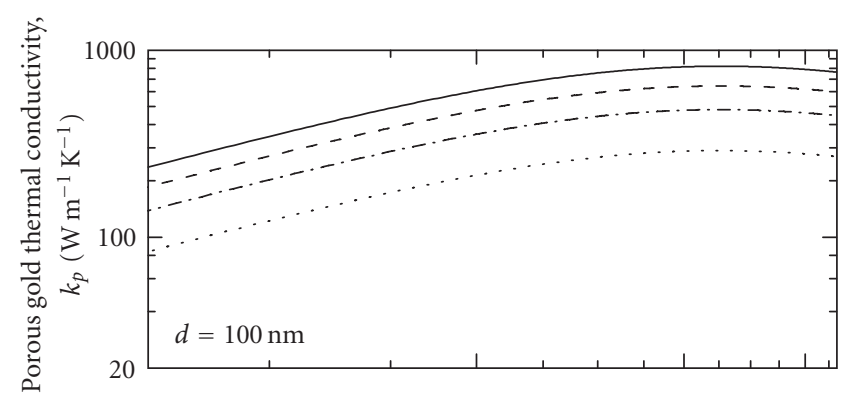

(b)

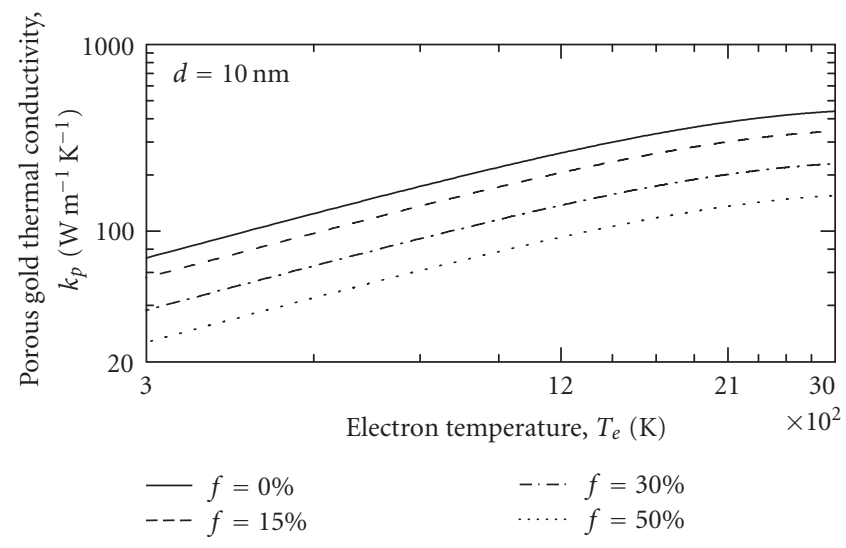

(c)

FIGURE 3: Thermal conductivity of porous gold material versus electron temperature for different porosities and different ligament thicknesses. A continued decrease in conductivity is observed with increasing porosity in addition to decreasing diameter, as expected from the expression for $k_{p}$ which is given by (2) and (3).

diameter and electron phonon coupling is given by using the thermal conductivity as defined by (2) and (3) in the TTM.

\section{EFFECTS ON ELECTRON-PHONON COUPLING MEASUREMENTS}

To examine this dependence, the TTR technique was used to measure the change in electron temperature during electronphonon coupling on a $2 \mu \mathrm{m}$ nanoporous Au film. Details of the TTR experimental setup are given by Hopkins and Norris [14]. The Au film was grown on an oxidized Si substrate by chemically dealloying a $40 \% \mathrm{Au}-60 \% \mathrm{Ag}$ composite with the fabrication processes outlined in Seker et al. [37]. The film used in this study was not actively annealed after the dealloying which resulted in a ligament thickness of about $100 \mathrm{~nm}$ estimated from the SEM micrograph, seen in Figure 4. Figure 5 shows a cross-sectional SEM micrograph of the porous Au sample. It is apparent in Figure 5 that the limiting dimension of the porous $\mathrm{Au}$ sample is the ligament diameter. This corresponds to a porosity, $f$, of about $35 \%$ [37]. The electron-temperature dependence after short pulsed laser heating on a $2 \mu \mathrm{m}$ nanoporous film with $35 \%$ porosity from TTM calculations is shown in Figure 6 for four different ligament thicknesses. Bulk thermophysical properties of $\mathrm{Au}$ were used in the calculations with an incident pump fluence of $10 \mathrm{~J} \mathrm{~m}^{-2}[14,38], 2.2 \times 10^{16} \mathrm{~W} \mathrm{~m}^{-3} \mathrm{~K}^{-1}$ was assumed for the electron-phonon coupling factor [12, 39], and an optical penetration depth of $12.5 \mathrm{~nm}$ was calculated from optical constants (The optical penetration depth of $\mathrm{Au}$ was calculated by the typical equation $\delta=\lambda /(4 \pi k)$, where $\lambda$ is the incident photon wavelength $(800 \mathrm{~nm})$ and $k$ is the complex part of the index of refraction of $\mathrm{Au}$ at $800 \mathrm{~nm}(k=5.125))$. The conductivity change from a wire diameter of $100 \mathrm{~nm}$ to $1 \mu \mathrm{m}$ is minimal, but still slightly observable. A greater change from $d$ of $100 \mathrm{~nm}$ to $10 \mathrm{~nm}$ to $1 \mathrm{~nm}$ is evident. This is expected due to the changes in thermal conductivity associated with $d$ reduction in $\mathrm{Au}$ shown in Figure 3. The TTM calculations were then fit to the experimental TTR data during the first 4.0 picoseconds after pulsed laser heating. The same assumptions and a similar fitting routine as Hopkins and Norris [14] were used, the difference being that the wire diameter, $d$, in the expression for thermal conductivity, was used as the fitting parameter. The results of the fit compared to the experimental data are shown in Figure 7. Seven TTR data scans were taken at different locations on the surface of the porous $\mathrm{Au}$ film, and the average best fit of the TTM was achieved with a wire diameter of $162 \mathrm{~nm}$ with a standard deviation of $11.3 \mathrm{~nm}$, which is in good agreement with the approximate ligament sizes observed in the SEM analysis. Using (3) (calculations of (3) are shown in Figure 3) and assuming $f=35 \%$ which was used in the TTM fit, the best fit wire diameter of $162 \mathrm{~nm}$ corresponds to a thermal conductivity of about $323 \mathrm{~W} \mathrm{~m}^{-1} \mathrm{~K}^{-1}$ assuming a maximum electron temperature of $820 \mathrm{~K}$ (from the TTM fit) and a cold lattice at $300 \mathrm{~K}$. This value of conductivity corresponds to overall thermal conductivity on the porous structure. For this situation $(d=162 \mathrm{~nm})$, the conductivity in each ligament is $622 \mathrm{~W} \mathrm{~m}^{-1} \mathrm{~K}^{-1}$ (see (2)), but since the probe in the TTR experiments measures the conductance averaged over a probe spot size of $\sim 10 \mu \mathrm{m}$ [14], the porous aspect of the sample which reduces the overall conductivity is observed. In a bulk Au sample, the thermal conductivity during this electron-phonon nonequilibrium predicted via (2) (when $v_{F} / d$ is negligible) is $733 \mathrm{~W} \mathrm{~m}^{-1} \mathrm{~K}^{-1}$.

\section{CONCLUSIONS}

In summary, the thermal conductivity in nanowires during electron-phonon nonequilibrium was studied and applied to $\mathrm{Au}$ nanoporous structures. Based on kinetic theory, a simple expression to predict the thermal conductivity in 


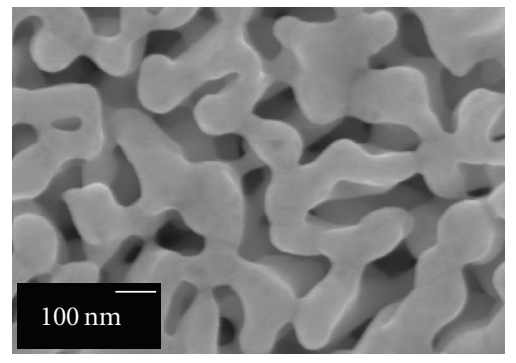

FIGURE 4: SEM micrograph of nanoporous $\mathrm{Au}$ sample used in this study. The ligament thickness is estimated as $100 \mathrm{~nm}$ and the porosity of about $35 \%$.

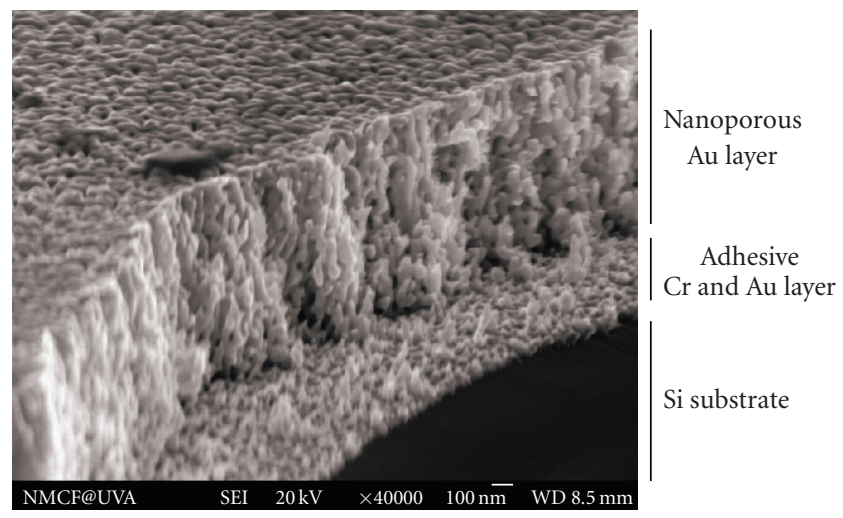

FIGURE 5: SEM micrograph of cross-section of porous Au sample showing the limiting dimension in each ligament is in the radial direction.

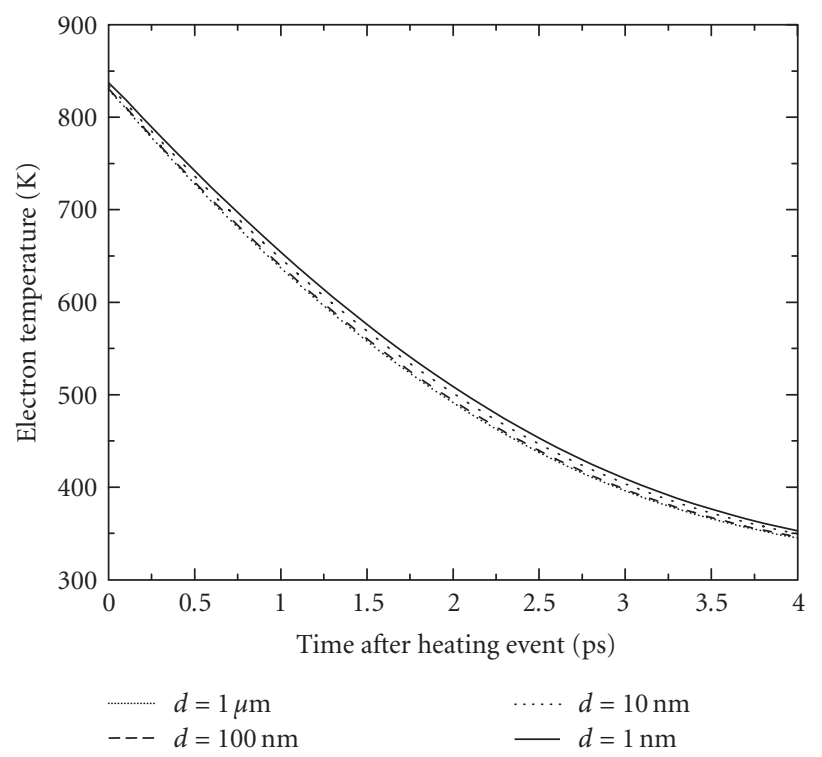

FIGURE 6: Change in electron temperature after short-pulsed laser heating in the event of electron-phonon nonequilibrium. Using the expression for $k_{p}$ and a porosity of $f=35 \%$, the sensitivity of electron cooling to ligament thickness and subsequent thermal conductivity is observed. It is apparent that electron-cooling is not extremely sensitive to the thermal conductivity reduction experienced during electron-phonon coupling.

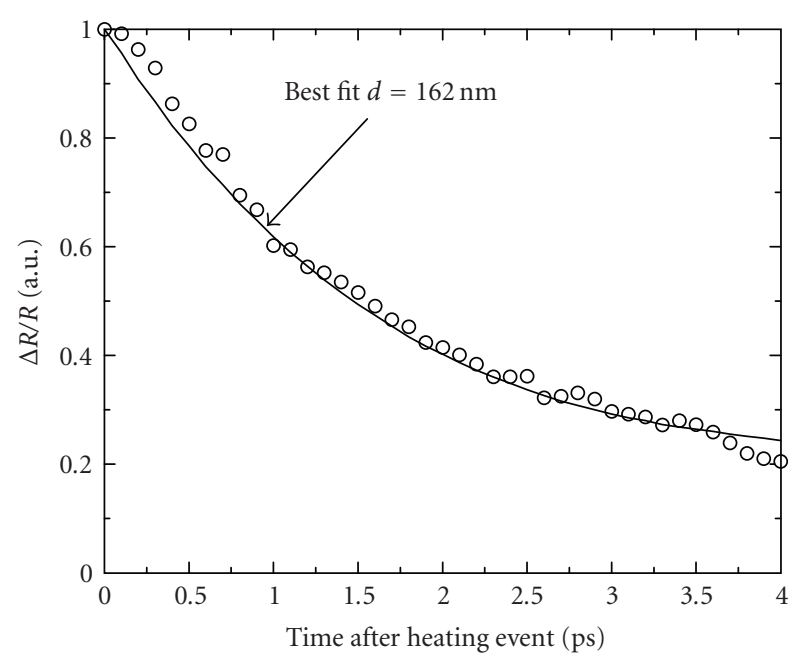

Figure 7: TTM using $k_{p}$ fit to TTR experimental data taken on a $2 \mu \mathrm{m} \mathrm{Au}$ nanoporous film with ligament thicknesses around $100 \mathrm{~nm}$. The fit was achieved by iterating $d$, the ligament, or "nanowire" thickness. A best fit was achieved with a thermal conductivity calculated using $d=162 \mathrm{~nm}$, in relatively good agreement with the ligament thickness of the nanoporous Au film that was tested.

nanowires was derived by taking into account electronboundary scattering. This expression agrees very well with results from other works. By introducing an expression to take into account the porosity of the nanocomposite, an expression for the thermal conductivity of the nanoporous Au was developed. This expression was then used in conjunction with the two-temperature model to study the change in electron temperature during short-pulsed laser heating. The TTM with $k_{p}$ was fit to transient thermoreflectance data taken on a nanoporous $\mathrm{Au}$ film with a best fit wire diameter that agrees with estimates of the wire diameter based on SEM micrographs. Therefore, the TTR technique can be used to characterize thermophysical properties of nanoporous materials and is sensitive to reductions in thermal conductivity that would arise due to the structure and geometry of nanoporous structures.

\section{ACKNOWLEDGMENTS}

The authors are greatly appreciative to W.-K. Lye at UVa for insight into nanoporous $\mathrm{Au}$ fabrication and G. Chen at MIT for helpful discussions on electron transport in nanowires. P. Hopkins was supported by the NSF Graduate Research Fellowship Program. Sandia is a multiprogram laboratory operated by Sandia Corporation, a LockheedMartin Company, for the United States Department of Energy's National Nuclear Security Administration under Contract DE-AC04-94AL85000.

\section{REFERENCES}

[1] N. Agrait, A. L. Yeyati, and J. M. van Ruitenbeek, "Quantum properties of atomic-sized conductors," Physics Reports, vol. 377, no. 2-3, pp. 81-279, 2003. 
[2] D. G. Cahill, W. K. Ford, K. E. Goodson, et al., "Nanoscale thermal transport," Journal of Applied Physics, vol. 93, no. 2, pp. 793-818, 2003.

[3] D. Stewart and P. M. Norris, "Size effects on the thermal conductivity of thin metallic wires: microscale implications," Nanoscale and Microscale Thermophysical Engineering, vol. 4, no. 2, pp. 89-101, 2000.

[4] A. Majumdar, K. Fushinobu, and K. Hijikata, "Effect of gate voltage on hot-electron and hot phonon interaction and transport in a submicrometer transistor," Journal of Applied Physics, vol. 77, no. 12, pp. 6686-6694, 1995.

[5] J. Erlebacher, M. J. Aziz, A. Karma, N. Dimitrov, and K. Sieradzki, "Evolution of nanoporosity in dealloying," Nature, vol. 410, no. 6827, pp. 450-453, 2001.

[6] J. Zhu, E. Seker, H. Bart-Smith, et al., "Mitigation of tensile failure in released nanoporous metal microstructures via thermal treatment," Applied Physics Letters, vol. 89, no. 13, Article ID 133104, 3 pages, 2006.

[7] Z. Liu and P. C. Searson, "Single nanoporous gold nanowire sensors," Journal of Physical Chemistry B, vol. 110, no. 9, pp. 4318-4322, 2006.

[8] P. M. Norris, A. P. Caffrey, R. J. Stevens, J. M. Klopf, J. T. McLeskey Jr., and A. N. Smith, "Femtosecond pump-probe nondestructive examination of materials," Review of Scientific Instruments, vol. 74, no. 1, pp. 400-406, 2003.

[9] S. I. Anisimov, B. L. Kapeliovich, and T. L. Perel'man, "Electron emission from metal surfaces exposed to ultrashort laser pulses," Soviet Physics - JETP, vol. 39, pp. 375-377, 1974.

[10] I. H. Chowdhury and X. Xu, "Heat transfer in femtosecond laser processing of metal," Numerical Heat Transfer, Part A, vol. 44, no. 3, pp. 219-232, 2003.

[11] G. L. Eesley, "Generation of nonequilibrium electron and lattice temperatures in copper by picosecond laser pulses," Physical Review B, vol. 33, no. 4, pp. 2144-2151, 1986.

[12] J. Hohlfeld, S.-S. Wellershoff, J. Güdde, U. Conrad, V. Jähnke, and E. Matthias, "Electron and lattice dynamics following optical excitation of metals," Chemical Physics, vol. 251, no. 1-3, pp. 237-258, 2000.

[13] P. E. Hopkins, J. M. Klopf, and P. M. Norris, "Influence of interband transitions on electron-phonon coupling measurements in Ni films," Applied Optics, vol. 46, no. 11, pp. 20762083, 2007.

[14] P. E. Hopkins and P. M. Norris, "Substrate influence in electron-phonon coupling measurements in thin Au films," Applied Surface Science, vol. 253, no. 15, pp. 6289-6294, 2007.

[15] J. L. Hostetler, A. N. Smith, D. M. Czajkowsky, and P. M. Norris, "Measurement of the electron-phonon coupling factor dependence on film thickness and grain size in $\mathrm{Au}, \mathrm{Cr}$, and $\mathrm{Al}$," Applied Optics, vol. 38, no. 16, pp. 3614-3620, 1999.

[16] T. Q. Qiu and C. L. Tien, "Size effects on nonequilibrium laser heating of metal films," Journal of Heat Transfer, vol. 115, pp. 842-847, 1993.

[17] T. Q. Qiu and C. L. Tien, "Heat transfer mechanisms during short-pulse laser heating of metals," Journal of Heat Transfer, vol. 115, pp. 835-841, 1993.

[18] A. N. Smith, J. L. Hostetler, and P. M. Norris, "Nonequilibrium heating in metal films: an analytical and numerical analysis," Numerical Heat Transfer, Part A, vol. 35, no. 8, pp. 859-873, 1999.

[19] D. S. Ivanov and L. V. Zhigilei, "Combined atomisticcontinuum modeling of short-pulse laser melting and disinte- gration of metal films," Physical Review B, vol. 68, no. 6, Article ID 064114, 22 pages, 2003.

[20] Z. Lin and L. V. Zhigilei, "Thermal excitation of d band electrons in Au: implications for laser-induced phase transformations," in High-Power Laser Ablation VI, vol. 6261 of Proceedings of SPIE, 62610U, pp. 1-14, Taos, NM, USA, May 2006.

[21] R. P. Wang, G. Xu, and P. Jin, "Size dependence of electronphonon coupling in $\mathrm{ZnO}$ nanowires," Physical Review B, vol. 69, no. 11, Article ID 113303, 4 pages, 2004.

[22] Y.-M. Lin, S. B. Cronin, J. Y. Ying, M. S. Dresselhaus, and J. P. Heremans, "Transport properties of Bi nanowire arrays," Applied Physics Letters, vol. 76, no. 26, pp. 3944-3946, 2000.

[23] E. Ramayya, D. Vasileska, S. M. Goodnick, and I. Knezevic, "Electron transport in Si nanowires," Journal of Physics: Conference Series, vol. 38, no. 1, pp. 126-129, 2006.

[24] A. N. Smith and P. M. Norris, "Numerical solution for the diffusion of high intensity, ultrashort laser pulses within metal films," in Proceedings of the 11th International Heat Transfer Conference (IHTC '98), vol. 5, pp. 241-246, Kyongju, Korea, August 1998.

[25] D. E. Gray, American Institute of Physics Handbook, McGraw Hill, New York, NY, USA, 1972.

[26] X. Y. Wang, D. M. Riffe, Y.-S. Lee, and M. C. Downer, "Timeresolved electron-temperature measurement in a highly excited gold target using femtosecond thermionic emission," Physical Review B, vol. 50, no. 11, pp. 8016-8019, 1994.

[27] A. H. MacDonald, "Electron-phonon enhancement of electron-electron scattering in Al," Physical Review Letters, vol. 44, no. 7, pp. 489-493, 1980.

[28] M. Kaveh and N. Wiser, "Electron-electron scattering in conducting materials," Advances in Physics, vol. 33, no. 4, pp. 257-372, 1984.

[29] N. W. Ashcroft and N. D. Mermin, Solid State Physics, Saunders College, Fort Worth, Tex, USA, 1976.

[30] J. M. Ziman, Electrons and Phonons, Clarendon Press, Oxford, UK, 1960.

[31] C. Y. Ho, R. W. Powell, and P. E. Liley, "Thermal conductivity of the elements," Journal of Physical and Chemical Reference Data, vol. 1, pp. 279-422, 1972.

[32] F. Cernuschi, S. Ahmaniemi, P. Vuoristo, and T. Mäntylä, "Modelling of thermal conductivity of porous materials: application to thick thermal barrier coatings," Journal of the European Ceramic Society, vol. 24, no. 9, pp. 2657-2667, 2004.

[33] R. M. Costescu, A. J. Bullen, G. Matamis, K. E. O’Hara, and D. G. Cahill, "Thermal conductivity and sound velocities of hydrogen-silsesquioxane low- $k$ dielectrics," Physical Review B, vol. 65, no. 9, Article ID 094205, 6 pages, 2002.

[34] D. S. McLachlan, "Equation for the conductivity of metalinsulator mixtures," Journal of Physics C, vol. 18, no. 9, pp. 1891-1897, 1985.

[35] D. A. G. Bruggeman, "Berechnung verschiedener physikalischer Konstanten von heterogenen Substanzen. I. Dielektrizitätskonstanten und Leitfähigkeiten der Mischkörper aus isotropen Substanzen," Annalen der Physik, vol. 416, no. 7, pp. 636-664, 1935.

[36] C. A. C. Bosco, A. Azevedo, and L. H. Acioli, "Substrate dependent ultrafast dynamics in thin NiFe films," Applied Physics Letters, vol. 83, no. 9, pp. 1767-1769, 2003.

[37] E. Seker, J. T. Gaskins, H. Bart-Smith, et al., "The effects of post-fabrication annealing on the mechanical properties 
of freestanding nanoporous gold structures," Acta Materialia, vol. 55, no. 14, pp. 4593-4602, 2007.

[38] F. Incropera and D. P. DeWitt, Fundamentals of Heat and Mass Transfer, John Wiley \& Sons, New York, NY, USA, 1996.

[39] A. N. Smith and P. M. Norris, "Influence of intraband transitions on the electron thermoreflectance response of metals," Applied Physics Letters, vol. 78, no. 9, pp. 1240-1242, 2001. 

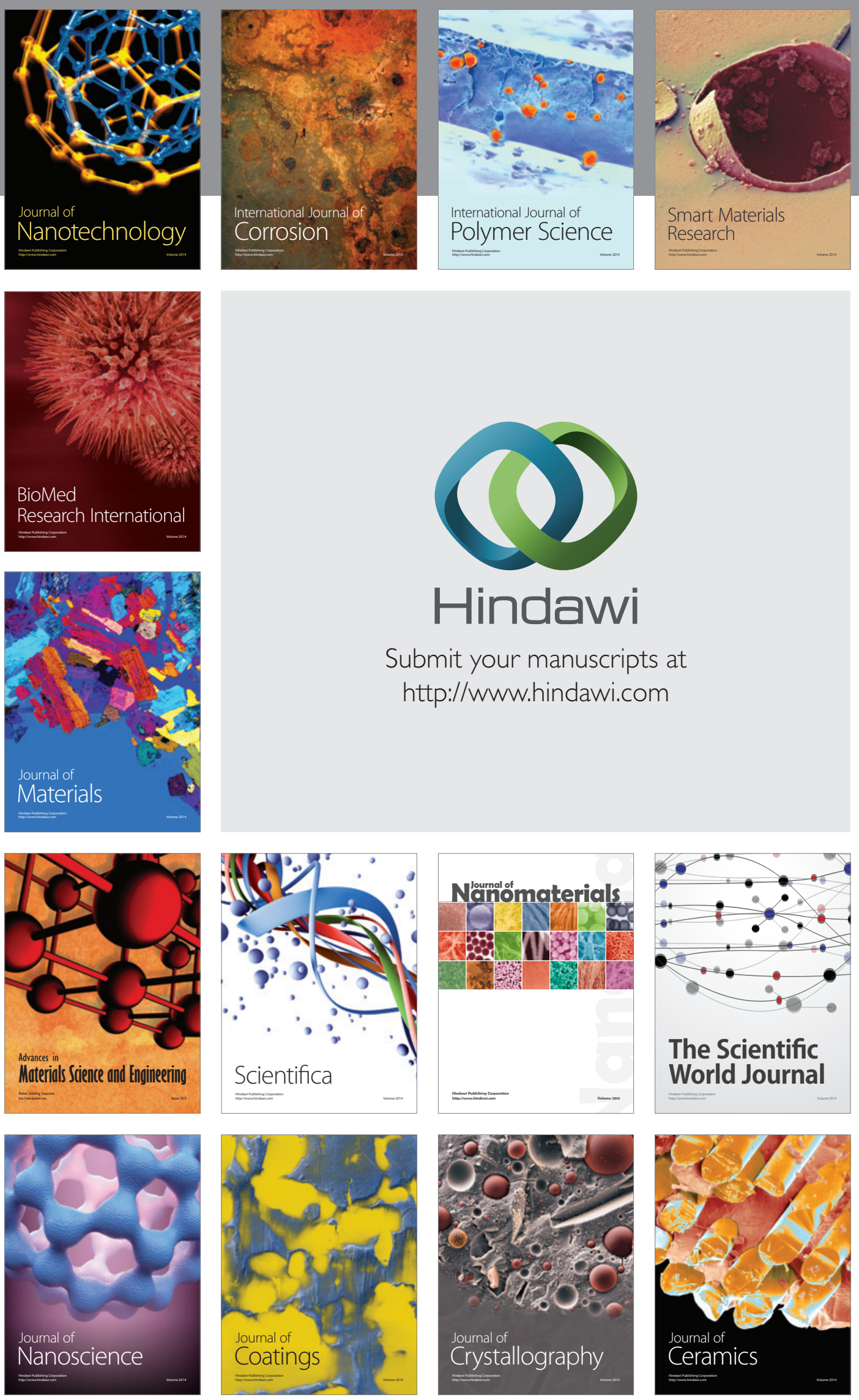

The Scientific World Journal

Submit your manuscripts at

http://www.hindawi.com

\section{World Journal}

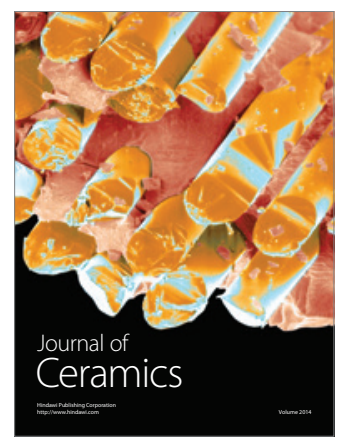

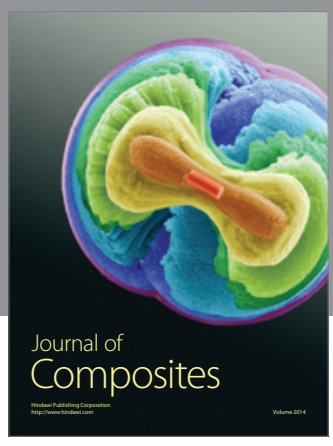
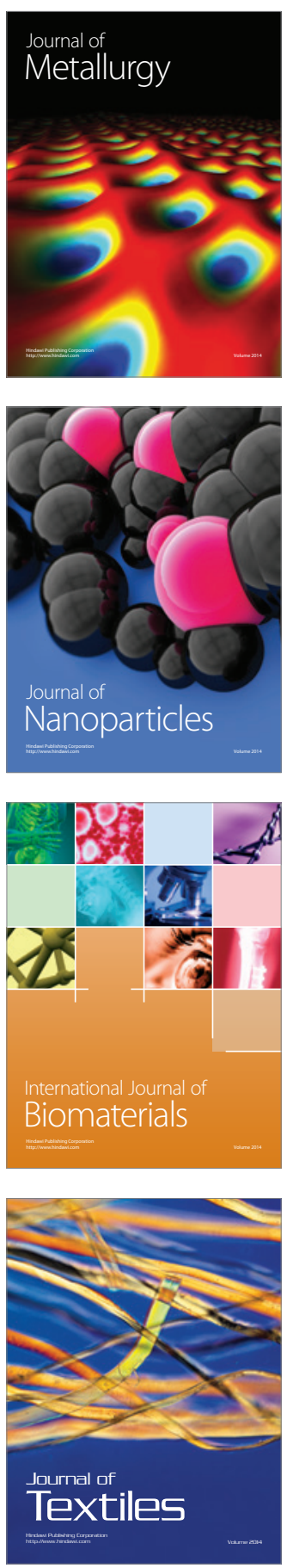